\title{
Pediatric Asthma in Southern Africa
}

\author{
Robin J. Green*
}

Department of Paediatrics and Child Health, University of Pretoria, South Africa

\begin{abstract}
Pediatric asthma has been well researched and actively managed in Southern Africa for many years. From the earliest study of asthma prevalence in 1979 revealing a significant rural - urban asthma gradient to more recent studies suggesting that asthma now abounds in all population and socio-economic groups, Southern Africa has been the subject of a significant number of publications relating to asthma prevalence, etiologies or associations and treatment guidelines. Asthma is now present in $20 \%$ of school-children across Southern Africa, but may not have the same etiological factors commonly seen in the West. Southern Africa has areas with a high asthma prevalence, areas with amongst the highest mortality in the world and similar problems to first-world countries with regard to morbidity and cost of disease. Guidelines for asthma management published from South Africa highlight the importance of aiming for complete asthma control and avoiding morbidity, exacerbations and mortality.
\end{abstract}

Key Words: Asthma, Pediatrics, Southern Africa.

\section{INTRODUCTION}

Paediatric asthma has been well researched and actively managed in Southern Africa for many years. The literature abounds with publications from this region on epidemiology, etiology or risk factors, clinical presentation, diagnosis and therapeutic strategies. This article will highlight many of the epidemiological and clinical considerations in asthma that have been published in Southern Africa in the last 30 years.

\section{PREVALENCE OF ASTHMA IN SOUTHERN AFRICA}

In 1969 Wesley et al. [1] published one of the earliest reports on childhood asthma in Africa, intimating that the condition was rare. They reported on asthma hospital admissions of black, Indian and white children in Durban, South Africa. During a 5-year period (1963-1967) these authors reported only 9 admissions for asthma among black children. This gave an admission rate of 0.02 percent (of all hospitalizations) compared with 0.79 percent for white children during the same time period. Although this was not a prevalence study, these figures do suggest that asthma in black and white communities was uncommon, relative to other causes for admission. The authors concluded that "true bronchial asthma as a reason for admission among Bantu children is excessively rare" and that "...it is probable that, as Bantu children become exposed to socio-economic environments similar to their white and Indian contemporaries, more will present with asthma".

The first Southern African study to verify the conclusions reached by Wesley et al. [1] regarding the influence of urbanization on asthma prevalence was performed in 1979 by van Niekerk and colleagues in the Cape Province [2]. They showed that the prevalence of exercise-induced bronchocon-

*Address correspondence to this author at the Department of Paediatrics and Child Health, University of Pretoria, South Africa; Tel: +27123545276; Fax:+27123545275; E-mail: Robin.Green@up.ac.za striction (EIB) in Xhosa children was 20 times higher in urban Guguletu than in rural Transkei. These investigators ensured that their two population groups were of the same tribe, one group having migrated to urban Guguletu near Cape Town, so the urban environment rather than genetic difference was implicated in the significantly higher prevalence of bronchial hyper-responsiveness.

In a follow up study one thousand four hundred and fiftyseven school children aged 10-14 years from the rural Transkei, from a recently urbanized peri-urban area and from urban Cape Town were studied using a questionnaire [3]. Four hundred and eighteen children had histamine challenges, and 492 tests for atopy were also conducted. As determined by bronchial challenge with histamine, $17 \%$ of rural and $34.4 \%$ of recently urbanized Xhosa children had increased BHR, a marked increase from the $0.03 \%$ and $3.17 \%$ prevalence of asthma previously found using exercise challenge. The prevalence of BHR in white urban children was $33 \%$. Sensitization to one or more aero-allergens, as indicated by ImmunoCAP RAST tests, was present in $36.6 \%$ of the rural Xhosa children with normal BHR and in $62.5 \%$ of those with increased BHR, a striking increase from that of previous studies. Atopic sensitization to one or more aeroallergens, as indicated by a skin prick test (SPT), was found in $42.3 \%$ of the recently urbanized Xhosa children and $45 \%$ of urbanized white children [3].

As no community prevalence study has been undertaken in black urban children in Soweto, figures for this region are unknown. However, impressions from medical colleagues working in the area are that asthma is increasing. Pediatric hospital admission records at Baragwanath Hospital for 1992 and 1993 revealed that of a total of 18044 admissions, 685, or $3.8 \%$, were for asthma. This constitutes almost a 200 -fold increase in paediatric admissions for asthma when compared with the figures previously presented by Wesley et al. [1], although, as mentioned, the 'denominator' may not have 
Table 1. Prevalence of Asthma in Southern Africa. A Summary of Studies

\begin{tabular}{|c|c|c|c|c|}
\hline & Age group & Cape Town & Polokwane & Maputo \\
\hline \hline $\begin{array}{c}\text { Current asthma } \\
\text { (mid-2000's) }\end{array}$ & $13-14$ year olds & $20.7 \%$ & $18 \%$ & $13.3 \%$ \\
\hline
\end{tabular}

been comparable between the studies. Nevertheless, it is probably reasonable to conclude from these latter two studies and the Baragwanath admission data, that urbanization (or urban dwelling) is a significant risk factor for BHR.

The question of race impacting on asthma prevalence was questioned by a study in Zimbabwe. The finding of Keeley et al., that the prevalence of BHR was similar in different race groups of the same socio-economic status, questions the race-based conclusions of previous authors [4]. On the other hand, Terblanche et al. found that the prevalence of exercise-induced bronchoconstriction was significantly higher among white children living in Cape Town $(5.87 \%)$ than in coloured children resident there (4.05\%). Importantly, they also commented on the low sensitivity $(0.31 \%)$ and positive predictive value $(46 \%)$ of the exercise test in diagnosing asthma at a community level [5].

Two further questionnaire-based epidemiological surveys were reported from Cape Town. In 1995 Ehrlich and colleagues [6] reported a prevalence of recent wheeze (previous 12 months) of $26 \%$, but asthma diagnosis of $10.8 \%$ [6], and in 1998 The International Study of Allergies and Asthma in Childhood (ISAAC) study reported a 12 month prevalence of self-reported asthma symptoms ranging from $7.5 \%$ to $17 \%$ (video questionnaire vs. written questionnaire) [7]. The prevalence of "asthma ever" was $13.3 \%$ in this group of children. The International Study of Asthma and Allergies in Childhood (ISAAC) programme was designed to determine the global epidemiology of childhood asthma. In this study [7] of 13- to 14-year-old children, only one South African centre, in Cape Town, participated in the first phase. The ISAAC Study was repeated in Phase III in 2005 and increases in asthma prevalence were noted (Table 1).

Calvert and Burney [8] confirmed the urban - rural difference $(8.7 \%$ vs. $14.5 \%$, respectively), and also demonstrated the increase in asthma prevalence over the 21 -year period from the previous Cape Study of 1979.

In two further epidemiological studies of asthma in children the prevalence was documented in two widely diverse areas of the country. In the Harbour Basin of Durban schoolchildren were subject to methacholine challenge testing and the prevalence of asthma was found to be a staggering 51.6\% [9]. This is an area of heavy industry and refineries and the atmospheric pollution from these factories may be the cause of this high statistic. In a further masters dissertation a study conducted in Thokoza, Gauteng the prevalence of EIB (using the 6 minute free running method) was found to be $7.26 \%$ (95\% confidence interval (CI): 4.5 - 10.3\%) in 475 nine and ten year old children [10]. This study also revealed findings suggestive of aetiology of asthma. The use of gas and electricity as domestic fuels was the strongest risk factor associated with EIB, as shown by logistical regression analysis using an adjusted odds ratio (OR) in a reduced model (OR 2.44, 95\% CI: $0.71-8.44$ and OR 2.33, 95\% CI: 0.59 - 9.24, respectively) [10].

The ISAAC protocol was applied in 27 schools in urban, suburban and semi-rural areas in Maputo, Mozambique [11]. A total of 2630 completed questionnaires were obtained from students aged 13-14 years, and 2383 from schoolchildren aged 6-7 years. The prevalence of current asthma was $13.3 \%$ in the two groups. Overall $35 \%$ of the adolescents and $23.8 \%$ of children were found to have at least one atopic condition or asthma in the last year. Exercise-induced wheeze, nocturnal cough and ever asthma were reported more frequently in children of the suburban area. These authors concluded that asthma and allergic diseases were important public health problems in Maputo and improvement in prevention and management plans were required [11].

Polokwane, in the north of South Africa, participated in the ISAAC protocol, documenting a prevalence of both asthma and allergic rhinitis in this area almost as high as in Cape Town [12]. The most important result of this study is that it confirmed a high ongoing prevalence of allergic conditions in the north of South Africa. This means that we can use this together with the data from Cape Town, to suggest a prevalence for all of South Africa. The prevalence of asthma symptoms in Polokwane was 18\%; not dissimilar to the $20,7 \%$ in Cape Town [12].

The prevalence, therefore, of asthma in Southern Africa is high and has increased in all populations over the last few decades.

\section{STUDIES SUGGESTING RISK FACTORS FOR PE- DIATRIC ASTHMA}

As the genetic pool of asthma cannot change rapidly over time, it seems logical that environmental factors are responsible for differences in asthma prevalences. Since Wesley et al. suggested that traditional rural life-styles protected against asthma and that urbanization thus promoted asthma development [1], many authors have speculated about mechanisms for the phenomenon.

Atmospheric pollution, dietary changes, changes in allergen load, improvements in health and hygiene (The Hygiene Hypothesis) and lifestyle changes have all been proposed for this phenomenon [13]. The Durban Harbour Basin Study [9] would suggest a strong case for atmospheric pollution while the findings of the Gauteng School Study [10] supports the Hygiene Hypothesis. In this study [10] it was found that children who lived in 'shacks' (ie. poorer socio-economic standards) were less likely to have asthma than their well-off peers. Studies in the Cape have suggested that changes in allergen load and atopic status mirror the rising prevalence of 
this condition. It seems clear that no one factor is responsible for asthma aetiology and that the condition is probably multi-factorial in aetiology as well as clinical expression.

Asthma has been linked to allergy in the western world. In a study in Soweto at least two-thirds of asthmatic children had a positive skin reaction to allergens [14]. The commonest allergens were grasses, pollen and house-dust mites. The high proportion of house-dust mite sensitivity (44.2\%) contradicted earlier beliefs that they are rare at higher altitudes [14]. The high proportion of atopy in asthmatics was confirmed in children from Johannesburg [15].

Despite the fact that 71.6 percent of the children at the Chris Hani Baragwanath Hospital in Soweto, South Africa had one or more positive skin prick tests (SPT) to common aero-allergens, they reported a low rate of positive family history for atopic conditions $(22.2 \%)$, suggesting that some new environmental exposure, rather than an inherited factor, was creating the allergic milieu in this group. A study was initiated to test this hypothesis [16]. Fifty four sets of parents and fifteen single mothers were studied. Of the forty eight atopic children thirty seven $(77 \%)$ had at least one parent with a positive SPT. Fifty five $\%$ of mothers had a positive SPT test while $48 \%$ of fathers had at least one positive SPT. The study proved that atopy was truly inherited but suggested that families with poor access to health care might not relate to common terms such as asthma. Of special interest in this study was the high prevalence of house dust mite SPT positivity amongst parents [16].

The Steinman Study [3] documented high allergy sensitivities in all children with asthma in the Cape, including sensitization to house dust mites in the rural Xhosa children for the first time. Passive cigarette smoking was not identified as a risk factor for increased BHR or atopy. Wood smoke in the indoor environment did not play a role in the rural Xhosa children's BHR. Ascaris infection does not appear to play any modifying role in the development of increased BHR in the rural or urban children. They found that increases in BHR in the rural and recently urbanized Xhosa children develop independently of increases in atopy. They concluded that 'our results challenge the 'hygiene' hypothesis as a complete explanation for the recent dramatic worldwide increases in allergic diseases [3].

A further study linked atopy and urbanization. The prevalence of EIB was higher in urban $(14.9 \%)$ than rural $(8.9 \%)$ areas $(\mathrm{P}<.0001)$. The difference in risk of EIB between urban and rural subjects was associated with atopy (OR for upper tertile of skin wheal diameter, $2.65 ; 95 \%$ confidence interval (CI), 1.43-4.89; $\mathrm{P}<.0001)$, increasing weight (OR for upper tertile of body mass index [BMI], 2.17; 95\% CI, 1.45-3.26; $\mathrm{P}=.001)$, and affluence [17].

With regard to environmental pollutants a number of epidemiological studies have been conducted in South Africa. In one study a total of 2,361 children from 17 schools were studied for asthma associations [18]. Meteorologically estimated exposure (MEE), but not simple distance from the refinery, was positively associated with having to take an inhaler to school (OR per interquartile range $1.22,95 \% \mathrm{CI}$ 1.06-1.40), and with a number of video elicited asthma symptoms, including recent waking with wheezing (OR
$1.33,95 \%$ CI 1.06-1.66) and frequent wheezing at rest (OR $1.27,95 \%$ CI 1.05-1.54). Symptom prevalences were higher than in other areas of the city, with frequent waking with wheezing being in great excess (OR 8.92, 95\% CI 4.7916.63) [19]. The results support the hypothesis of an increased prevalence of asthma symptoms among children in the area as a result of refinery emissions and provide a substantive basis for community concern. In further studies, predictors of asthma/wheeze were hay fever (OR 5.30; 95\% CI 3.16-8.89), eczema (OR 2.19; 95\% CI 1.33-3.62), parental asthma (OR 1.77; 95\% CI 1.11-2.84), absence of paternal contribution to income (OR 1.72; 95\% CI 1.17-2.54), maternal smoking in pregnancy (OR 1.87; 95\% CI 1.25-2.81), and each additional household smoker (OR 1.15; 95\% CI 1.011.30). Findings were similar, with higher odds ratios for most variables, except number of household smokers, when the group was restricted to children with parent-reported asthma [19].

Two studies have emanated from Polokwane in an attempt to define asthma and risk factors for asthma in that region. In the first published study the aim was to assess the 12-month prevalence of wheeze and severe wheeze together with their risk factors [20]. This was a questionnaire study (within the context of ISAAC) and the findings were of a $11.2 \%$ prevalence of wheeze and $5.7 \%$ prevalence of severe wheeze in these children. In addition these authors report that living in a rural area significantly decreased the likelihood of wheeze (by 31\%) while exposure to environmental tobacco smoke at home and the presence of both eczema and rhinoconjunctivitis symptoms increased the likelihood of wheeze. Rhinoconjunctivitis was also a predictor of severe wheeze. In their conclusion the authors suggest that wheeze (and probably therefore asthma) is an emerging public health problem in Polokwane [20].

Also published in 2009 was a study of determinants of asthma at the district, school and individual level in Polokwane [21]. 742 school children were questioned. The authors report that persistent cough, exposure to smoke at home and the lack of access to a flushing toilet are key predictors of asthma in children. These findings deserve our attention as we know that asthma in South Africa has a distinct pattern that is not identical to atopic asthma seen elsewhere in the world. We urgently need studies such as these to explore our local conditions that expose asthma phenotypes.

A few studies have looked at the relationship between infectious diseases and asthma. In a Cape Town study of unselected urban Black African high school children, SPT's were positive for aeroallergens in $32.3 \%$ of subjects [22]. Thirty four percent had elevated ascaris IgE. Total IgE was higher in atopic compared to non-atopic subjects and correlated with the number of positive skin prick tests, self-reported asthma and BHR. Subjects without allergy (or) atopy had a median total $\mathrm{IgE}$ of $80-90 \mathrm{kU} / \mathrm{I}$. In addition total IgE correlated with ascaris IgE. Subjects with no ascaris sensitization had median total $\mathrm{IgE}$ of $77.1 \mathrm{kU} / 1$. Subjects with neither atopy/asthma nor ascaris sensitization had a median total $\operatorname{IgE}$ of $69.9 \mathrm{kU} / \mathrm{I}$, similar to the levels seen in people of other genetic origins. This study suggests that helminthic infection rather than genetic differences, may be the major determining factor of $\mathrm{IgE}$ levels in certain populations [22]. 
Table 2. Risk Factors Identified in Southern Africa that may be Linked to Increases in Asthma Prevalence

\begin{tabular}{|c|c|c|}
\hline Risk Factor & Risk & References \\
\hline Atopy & $\begin{array}{l}71.6 \% \text { of asthmatics } \\
\text { OR } 2.65 \\
\text { OR } 2.69 \text { risk in urban vs rural }\end{array}$ & $\begin{array}{l}\text { Luyt DK [14] } \\
\text { Green RJ [15] } \\
\text { Steinman H [3] } \\
\text { Calvert J [17] }\end{array}$ \\
\hline Ascaris & $\begin{array}{l}\text { Helminthic infection determines atopy } \\
\text { Helminthic infection protects against } \\
\text { asthma }\end{array}$ & $\begin{array}{l}\text { Levin M [22] } \\
\text { Obihara C [23] }\end{array}$ \\
\hline Lack of MTB & OR 0.43 (allergic rhinitis) & Obihara C [24] \\
\hline Not breast feeding & Risk in non-allergic families & Obihara C [25] \\
\hline
\end{tabular}

However a previous study had suggested that immune responses to Ascaris (Ascaris-sIgE) may be a risk factor of atopic disease in populations exposed to mild Ascaris infection and that Mycobactrium tuberculosis infection may be protective against this risk, probably by stimulation of antiinflammatory networks [23].

Obihara conducted a subsequent study to confirm the inverse relationship between Mycobacterium tuberculosis (MTB) and asthma. Children with positive Tine Skin Test (TST) $(>$ or $=10 \mathrm{~mm}$ ) were significantly less likely to have allergic disease symptoms, in particular allergic rhinitis (adjusted OR $0.43 ; 95 \%$ CI $0.24-0.79$ ) than those with negative TST [24]. There was a significant inverse linear trend in the relation of TST size in millimetre and the frequency of allergic disease symptoms. These results of inverse association of strong TST reaction and allergic disease symptoms in children from a TB endemic area are in support of the hypotheses that allergic inflammation may be inhibited by chronic infections, such as MTB [24].

The fact that breastfeeding may protect against allergic disease remains controversial even in the developed world. There is one report from South Africa [25]. Data was collected from a $15 \%$ random sample of households from two poor suburbs of Cape Town, South Africa. Out of the 861 children included in the study, allergic disease in general, and hay fever in particular, were significantly less frequent in those with prolonged ( $>$ or $=6$ months) breastfeeding. There was a significant linear inverse association between breastfeeding duration and allergic disease in children without allergic parents, but not in children with an allergic predisposition. In conclusion, these results from a developing country suggest a protective effect of prolonged breastfeed- ing on the development of allergic disease, particularly hay fever, in children born to nonallergic parents. This protective effect was not found in children with an allergic predisposition [25].

All these risk factors are summarized in Table 2.

Recent literature has suggested a close link between viral infections, atopy and asthma especially with regard to risk for asthma. Unfortunately no data is available from Southern Africa to confirm or refute this proposition.

\section{DIAGNOSIS OF ASTHMA}

Diagnosis of asthma is frequently centered on recurrent wheeze but at least one South African Pediatric study has suggested cough is important [26]. Of the 468 (297 boys) asthmatics studied, $456(97.4 \%)$ presented with cough, 362 $(77.3 \%)$ with wheeze, $286(61.1 \%)$ with a tight chest and $277(59.2 \%)$ with breathlessness. Cough as sole symptom occurred in $102(21.8 \%)$ while only $8(1.7 \%)$ wheezed and did not cough. Commonest symptom triggers were upper respiratory tract infections and activity/exercise. An individual atopic background was common - allergic rhinitis in 413 $(88.2 \%)$ - as was a family history of atopy, present in 390 $(83.3 \%)$. This study also suggested that delay in asthma diagnosis is common. Prolonged symptomatic periods occurred in most patients before asthma was diagnosed (among children diagnosed after the age of 4 years, $50 \%$ had been symptomatic for more than 3 years). 'Delay to diagnosis' was not influenced by presenting symptoms or by previous hospitalization for asthma. Other respiratory diagnoses of bronchitis and pneumonia were common, possibly because of misdiagnosis [15]. 
South African Asthma Guidelines for asthma diagnosis and management have been published for the last 15 years and the latest Guidelines provide important insights into asthma diagnosis and management [26].

\section{Asthma in Young Children}

A simple clinical index (asthma predictive index) based on the presence of a wheeze before the age of 3, and the presence of 1 major risk factor (parental history of asthma or eczema) or 2 of 3 minor risk factors (eosinophilia, wheezing without colds, and allergic rhinitis), has been shown to predict the occurrence of asthma in later childhood in children in developed countries [27]. While useful to diagnose asthma in large epidemiological studies in Europe and America (Evidence B), this finding has not been applied in the context of African children, who have less atopy. A recent publication categorized wheeze in young children into 2 broad groups: episodic (viral) wheeze and multi-trigger wheeze [28]. The group of children who have multi-trigger wheeze have symptoms precipitated by factors other than viral infections (such as allergens and exercise) and are likely to be asthmatic. The diagnosis of preschool asthma should be based on a composite set of diagnostic criteria and, while no tests diagnose asthma with certainty in young children, the features listed in Table $\mathbf{3}$ may be considered as useful adjuncts in making a diagnostic decision.

Table 3. Features Suggestive of Asthma in Children $<5$ Years Old

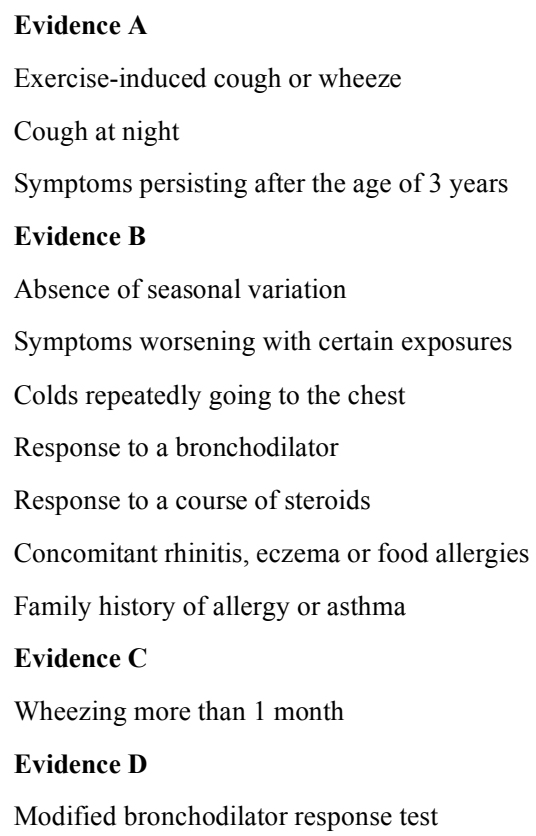

\section{Children Older than 5 Years}

A careful history and physical examination, together with objective evidence of reversible airflow obstruction after administration of a short-acting $\beta 2$-agonist (SABA) (an increase in forced expiratory volume in 1 second (FEV1) $>12 \%$ or in peak expiratory flow rate (PEF) $>15 \%$ after 10 minutes), will in most instances confirm the diagnosis. Monitoring symptoms and PEF using a diary card is also useful for making the diagnosis; diurnal PEF variability $>20 \%$ is highly suggestive of asthma.

\section{TREATMENT OF ASTHMA [26]}

\section{Goals and Principles of Management}

\section{Goals}

The goal of asthma therapy is asthma control [29]. Control implies that the asthmatic child is able to lead a normal and physically active life. The criteria for 'normal life' are to:

- be completely free from any symptoms, i.e. cough, wheeze and breathlessness

- attend school regularly and participate fully in all school activities, including sport

- sleep restfully, free from night-time cough and/or wheeze

- grow and develop normally

- minimise the number of attacks of acute asthma and avoid hospitalization

- avoid or minimize medication-related side-effects.

\section{Principles}

A comprehensive therapeutic approach is required to meet the above objectives, which includes the following:

- early diagnosis and objective assessment of severity

- control of the environment to exclude cigarette smoke and reduce exposure to triggers such as viral infection and allergens

- optimal use of medications to limit side-effects and cost, using the most appropriate delivery system

- follow-up and regular re-evaluation (clinical evaluation and quality of life)

- patient and parent education.

\section{Environmental Control}

Avoidance of allergens (based on history and allergy testing) and pollutants is recommended when there is a clear association between exposure and symptoms.

The principles of pharmacotherapy are available from most Guidelines.

\section{Patient Education for Asthma}

There is no doubt that Southern Africans with asthma need special attention with regard to education. Levin has shown that local language and culture are important when educating asthmatics [30]. A study in Mozambique of 152 adults accompanying asthmatic children to consultations in the Paediatrics department of Maputo Central Hospital (Mozambique) found that knowledge about asthma was poor: $11 \%$ of the adults thought that asthma was contagious and transmitted from person to person, and 4\% thought that it was transmitted by contaminated food. More than half the parents thought that the child could not lead a normal life even during the periods between attacks [31]. A large pro- 
portion of the parents thought that asthma could be cured by medical treatment (in 7\% of cases) and by alternative treatment (in $43 \%$ of cases). The precipitating factors were well identified by the adults, but they had an inaccurate perception of the symptoms of an asthma attack. The actions of the various classes of drugs used were poorly understood. Education programs for both the children and their parents should be developed to improve the management of asthmatic children. Surveys of this type facilitate the targeting of such programs.

A great deal of fear and ignorance surrounds asthma, so a real need exists for a greater level of patient education. This study highlights how much it is needed in the rural areas of Southern Africa. Nurses currently play a far greater role than doctors in asthma management and education, particularly in rural clinics. Limitation of available drugs, incorrect dosage formulations and sub-optimal prophylaxis are of concern. Use of the inhaled prophylactic therapy in only $30 \%$ of patients (although $77.4 \%$ indicate that asthma impacts on their quality of life), highlights mismanagement, and suggests that isolated symptom reporting is a poor indication of overall well-being or disease control. Asthmatics themselves need to have the specific symptoms of poor control (wheeze and cough) spelt out, as this increases their awareness (versus the use of the general term "frequency of symptoms") Ninety-six percent of children and $91 \%$ of adult respondents admitted having being treated by a traditional healer (witchdoctor/sangoma - employing herbal and non-medicinal therapies) [31].

A similar finding of poor asthma control was obtained from rural South Africa [32].

\section{MORBIDITY FROM ASTHMA IN SOUTH AFRICA}

Despite availability of useful asthma therapies and treatment strategies the morbidity from asthma remains significant. The medical or clinical effects and treatment of the disease are very well known but less well known, and certainly seldom discussed, are the social and psychological consequences of asthma as a chronic illness.

In 2000 a project was launched to collect data on the status of asthmatic patients in South Africa [33]. Questionnaires were distributed randomly to diagnosed asthmatics in doctors consulting rooms and at pharmacies. 710 respondents met the criteria for analysis, ie. had asthma, presently on medication. Symptom analysis reveals that most patients still had symptoms of asthma; cough and wheeze. Symptoms were exacerbated by exercise in nearly two-thirds of respondents. Daily activities caused symptom exacerbation in $46.2 \%$. Nocturnal wakening due to asthma occurred frequently with $36.9 \%$ of respondents being woken more than 4 times per week.

With regard to quality of life certain pertinent responses were received. Only $35.1 \%$ of respondents had not missed school or work in the preceding year and $26.3 \%$ had missed 1-3 days. Lastly, the rate of hospital admission and crisis control visits were recorded. $15.9 \%$ of responders had been admitted to hospital in the previous year, and doctor-calledout, doctor visited; or casualty visited in $13.9 \%, 27.3 \%$ and $24.8 \%$ respectively.
This symptom exacerbation as well as acute attacks and doctor crisis consultation or hospitalization at the rates indicated, not only supports the notion of poor therapy (or possibly poor compliance) but also has then an enormous impact on the health-related quality of life of South Africa's asthmatics. Even the attempt to pass this problem off as one of compliance being poor, is a failure on behalf of the medical fraternity to educate patients completely.

\section{PREVALENCE OF SEVERE ASTHMA IN SOUTH AFRICA}

In two recent surveys of school children, utilising international methodology that permits comparisons with surveys performed in other countries (ISAAC surveys) the prevalence of asthma was similar to the global average of approximately $12 \%$ amongst children aged 6 to 11 , and South Africa ranked $25^{\text {th }}$ among the 93 countries surveyed [34]. More alarming however was the fact that in surveys of asthma mortality, South Africa ranked $3^{\text {rd }}$ or $4^{\text {th }}$ highest in the world, whether expressed as deaths as a percentage of the total population or of the asthma population [7]. In further analysis of the Cape Town ISAAC data, it was demonstrated that while asthma was more prevalent in affluent households, the burden of morbidity and mortality was in households with low socioeconomic status [35]. Although many factors operating in impoverished communities contribute to the suboptimal care of children with chronic disease like asthma, access to and the availability of controller medication remains the most important preventable factor. There is an urgent requirement for increasing awareness and vigilance in the diagnosis of childhood asthma and for the use of effective controller regimens with long-term monitoring and follow-up. Although inhaled corticosteroids form part of the essential drug list and are available in most community health centres, physicians and patients report prescriber compliance with guidelines and erratic drug supply as a causes of unnecessary poor treatment results. This probably accounts for the persistently high mortality when compared with the falling rates in other parts.

In South Africa it is estimated that most asthmatics (approximately $80 \%$ ) have mild disease, and that only $5-6 \%$ of adult asthmatics have severe or very severe disease. In a South African study [36] of 411 patients cared for by a large medical aid, $41 \%$ were classified as having mild asthma, $49 \%$ moderate, and $10 \%$ severe. In this study, $33 \%$ of severe asthmatics required hospitalization compared to $2 \%$ of mild asthmatics.

Life-threatening attacks of asthma requiring intensive care unit (ICU) management at Red Cross War Memorial Children's Hospital in Cape Town were noted to occur in some patients in the same or adjacent months of different years [37]. A retrospective case-controlled study was performed of 21 such 'seasonal' patients who presented to the ICU over a 14 -year period. The group made up $6.5 \%$ of all asthma patients admitted to the ICU and their 65 admissions made up $15.6 \%$ of all ICU asthma admissions during this period. The control group consisted of patients with recurrent admissions that occurred in 'random' months. The two groups were compared in respect of demographic and clinical data. Patients requiring seasonal admissions were shown to form a distinct sub-population of children with severe 
asthma, some with a family history of fatal asthma, who were less likely to 'outgrow' asthma in childhood, were more likely to require maintenance steroid therapy for asthma management, and significantly more often had positive radioallergosorbent tests to Aspergillus and Cladosporium sp. and to grass pollen. A retrospective analysis of dates of severe asthma attacks may identify individual seasonality, which is a risk factor for life-threatening and intractable asthma.

\section{COST OF ASTHMA IN SOUTH AFRICA}

A study performed in the Transkei in 1993 revealed asthma treatment cost to be only $0.38 \%$ of that country's total annual medical expenditure [38]. The average hospital stay for an asthma exacerbation was 9 days, and it seems that the indirect costs of asthma would be significant. Interestingly it was demonstrated that the annual cost of beclomethasone dipropionate (BDP) for 1 person at that time equated to the cost of 2.25 days in hospital, yet only 43 prescriptions for BDP were filled in the Transkei in 1992! Indirect costs of asthma and costs associated with uncontrolled disease dominate the cost structure for asthma in South Africa. The appropriate costs of medication, irrespective of their absolute cost, are but the tip of the cost iceberg in asthma.

However we view the costs of asthma, both hidden and obvious, there is no question that only by attention to the subtleties of this cost equation will we begin to address the important impact asthma has on both the quality of life of sufferers and health budget of this country.

\section{CONCLUSION}

Southern Africa poses a number of questions in the asthma arena that deserve our attention. It seems we have areas with a high asthma prevalence. We also have areas with amongst the highest mortality in the world. We have similar problems to first-world countries with regard to morbidity and cost of disease. Putting all of these phenomena together may suggest a number of intervention strategies. However, the overwhelming message that these figures suggest is that to date intervention programmes to fight asthma have failed and that probably the most important reason for declining mortality from asthma around the world and in many parts of South Africa is not better understanding and treatment of chronic asthma but rather better access to emergency medical care for acute asthma. This is a worrying sign and needs the urgent attention of asthma education programmes.

Finally our attention in the management of asthma, especially chronic asthma, should be on achieving better control of the disease through aiming to meet the 'Goals of Asthma Management' as set out in Guidelines. To this end it seems reasonable that our objectives in this respect have been a dismal failure. Today we know that nothing short of 'Total Asthma Control' is both possible and acceptable.

\section{REFERENCES}

[1] Wesley AG, Clyde JH, Wallace HL. Asthma in Durban children of three racial groups. S Afr Med J 1969: 87-89.
[2] van Niekerk CH, Weinberg EG, Shore SC, et al. Prevalence of asthma: a comparative study of urban and rural Xhosa children. Clin Allergy 1979; 9: 319-324.

[3] Steinman HA, Donson H, Kawalski M, Toerien A, Potter PC. Bronchial hyper-responsiveness and atopy in urban, peri-urban and rural South African children. Pediatr Allergy Immunol 2003; 14 : 383-93.

[4] Keeley DJ, Neill P, Gallivan S. Comparison of the prevalence of reversible airways obstruction in rural and urban Zimbabwean children. Thorax 1991; 146: 549-553.

[5] Terblanche E, Stewart RI. The prevalence of exercise-induced bronchoconstriction in Cape Town school children. S Afr Med J 1990; 78: 744-7.

[6] Ehrlich RI, du Toit D, Jordaan E, Volmink JA, Weinberg EG, Zwarenstein M. Prevalence and reliability of asthma symptoms in primary school children in Cape Town. Int J Epidemiol 1995; 24: 1138-1145.

[7] ISAAC Steering Committee. Worldwide variation in prevalence of symptoms of asthma, allergic rhinoconjunctivitis and atopic eczema. ISAAC. Lancet 1998; 351: 1225-1232.

[8] Calvert JM, Burney PGJ. Exercise induced bronchospasm and positive skin prick tests are commoner in urban vs rural African children (poster). $97^{\text {th }}$ International Conference of the American Thoracic Society, San Francisco, 18-23 May 2001.

[9] Jack CB. Asthma in KwaZulu Natal. S Afr Resp J 2006; 12: 14-17.

[10] Mashalane MB, Stewart A, Feldman C, Becker P, de Charmoy S. Prevalence of exercise-induced bronchospasm in Thokoza schoolchildren. S Afr Med J 2006; 96: 67-70.

[11] Mavale-Manuel S, Joaquim O, Macome C, et al. Asthma and allergies in school children of Maputo. Allergy 2007; 62: 265-71.

[12] Ait-Khaled N, Odhiambo J, Pearce N, et al. Prevalence of symptoms of asthma, rhinitis and eczema in 13- to 14-year old children in Africa: the International Study of Asthma and Allergies in Childhood Phase III. Allergy 2007; 62: 247-58.

[13] Martinez FD. The coming- of- age of the hygiene hypothesis. Respir Res 2001; 2: 129-132.

[14] Luyt DK, Davis G, Dance M, Simmank K, Patel D. Clinical characteristics of black asthmatic children. S Afr Med J 1995; 85: 9991001

[15] Green R, Luyt D. Clinical characteristics of childhood asthmatics in Johannesburg. S Afr Med J 1997; 87: 878-82.

[16] Davis G, Green RJ, Hon H. Understanding the concept of 'family history' in black asthmatic children. S Afr J Child Health 2007; 1: 14-18.

[17] Calvert J, Burney P. Effect of body mass on exercise-induced bronchospasm and atopy in African children. J Allergy Clin Immunol 2005; 116: 773-9.

[18] White N, teWaterNaude J, van der Walt A, Ravenscroft G, Roberts W, Ehrlich R. Meteorologically estimated exposure but not distance predicts asthma symptoms in school children in the environs of a petrochemical refinery: a cross-sectional study. Environ Health 2009; 8: 45.

[19] Ehrlich RI, Du Toit D, Jordaan E, et al. Risk factors for childhood asthma and wheezing. Importance of maternal and household smoking. Am J Respir Crit Care Med 1996; 154(Pt 1): 681-8.

[20] Wichmann J, Wolvaardt JE, Maritz C, Voyi KV. Household conditions, eczema symptoms and rhinitis symptoms: relationship with wheeze and severe wheeze in children living in the Polokwane area. S Afr Matern Child Health J 2009; 13: 107-18.

[21] Maluleke KR, Worku Z. Environmental determinants of asthma among school children aged 13-14 in and around Polokwane, Limpopo Province, South Africa. Int J Environ Res Public Health 2009; 6: 2354-74.

[22] Levin ME, Le Souëf PN, Motala C. Total IgE in urban Black South African teenagers: the influence of atopy and helminth infection. Pediatr Allergy Immunol 2008; 19: 449-54.

[23] Obihara CC, Beyers N, Gie RP, et al. Respiratory atopic disease, Ascaris-immunoglobulin $\mathrm{E}$ and tuberculin testing in urban South African children. Clin Exp Allergy 2006; 36: 640-8.

[24] Obihara CC, Kimpen JL, Gie RP, et al. Mycobacterium tuberculosis infection may protect against allergy in a tuberculosis endemic area. Clin Exp Allergy 2006; 36: 70-6. 
[25] Obihara CC, Marais BJ, Gie RP, et al. The association of prolonged breastfeeding and allergic disease in poor urban children. Eur Respir J 2005; 25: 970-7.

[26] Motala C, Green RJ, Manjra A, Potter PC, Zar HJ. Guideline for the management of chronic asthma in children - 2009 update. S Afr Med J 2009; 99: 877-912.

[27] Castro-Rodriguez JA, Holberg CJ, Wright AL, Martinez FD. A clinical index to define risk of asthma in young children with recurrent wheezing. Am J Respir Crit Care Med 2000; 162(4 Pt 1): 1403-6.

[28] Brand PLP, Baraldi E, Bisgaard H, et al. Definition, assessment and treatment of wheezing disorders in preschool children: an evidence-based approach. Eur Respir J 2008; 32: 1096-1110.

[29] Global Initiative for Asthma. Global Strategy for Asthma Management and Prevention. NHLBI/WHO Workshop Report. National Institutes of Health. National Heart, Lung, and Blood Institute. Publication Number 95-3659, 1996.

[30] Levin ME. Impact of language and culture on the quality of medical communication and care. Curr Allergy Clin Immunol 2007; 20(2): 54 .
[31] Mavale-Manuel S, Duarte N, Alexandre F, et al. Knowledge, attitudes, and behavior of the parents of asthmatic children in Maputo. J Asthma 2004; 41: 533-80.

[32] Green RJ, Greenblatt M, Plit M, Jones S, Adam B. Asthma management and perceptions in rural South Africa. Ann Allergy Asthma Immunol 2001; 86: 343-347.

[33] Green RJ, Davis G, Price D. Perceptions, impact and management of asthma in South Africa: a patient questionnaire study. Primary Care Respir J 2008; 17: 212-6.

[34] Beasley R. The Global Burden of Asthma Report, Global Initiative for Asthma (GINA). Available from http://wwwginasthmaorg 2004.

[35] Zar H. Fatal and near-fatal asthma in South Africa. Curr Allergy Clin Immunol 2004; 17: 56-58.

[36] Burns DG. Third Triennial World Asthma Meeting 2001 (Poster).

[37] Roux P, Smit M, Weinberg EG. Seasonal and recurrent intensive care unit admissions for acute severe asthma in children. S Afr Med J 1993; 83(3): 177-9.

[38] Kling S. Asthma cost and prevalence in South Africa. Allergy Society of South Africa Congress 2003 (Oral Presentation).

(C) Robin J. Green; Licensee Bentham Open.

This is an open access article licensed under the terms of the Creative Commons Attribution Non-Commercial License (http://creativecommons.org/licenses/by-nc/3.0/) which permits unrestricted, non-commercial use, distribution and reproduction in any medium, provided the work is properly cited. 